\title{
Comportamento de chuvas intensas para bacia em região semiárida de Caatinga preservada
}

\author{
Gláuber Pontes Rodrigues ${ }^{1} \oplus$, José Carlos de Araújo이 \\ ${ }^{1}$ Universidade Federal do Ceará, Programa de Pós-Graduação em Engenharia Agrícola, Fortaleza, CE, Brasil. E-mail: pontesglauber@gmail.com; jcaraujo@ufc.br
}

RESUMO: Conhecer o comportamento das chuvas intensas é fundamental para planejamento de bacias hidrográficas, contudo demanda robustos bancos de dados pluviográficos, ainda escassos na região semiárida brasileira. $O$ objetivo deste trabalho foi avaliar as curvas intensidade - duração - frequência (IDF) na Bacia Experimental de Aiuaba, Ceará. Com base em medições a cada $5 \mathrm{~min}$ durante 16 anos (2003 a 2018) foram geradas curvas IDF para os períodos de $5 \mathrm{~min}, 30 \mathrm{~min}, 1 \mathrm{~h}, 2 \mathrm{~h}, 3 \mathrm{~h}, 6 \mathrm{~h}, 12 \mathrm{~h}, 18 \mathrm{~h}$ e $24 \mathrm{~h}$ e calibrados os parâmetros da equação de Otto Pfafstetter para as curvas IDF da referida bacia. Da investigação pôde-se concluir que: as curvas IDF (inéditas para a região dos Inhamuns, CE) são compatíveis com os de outros estudos em regiões semiáridas e indicaram ser uma propriedade macrorregional; a erosividade indicou um valor máximo para chuvas de duração igual a 3 horas, sendo esse um efeito combinado de alta intensidade e alta energia cinética. Esta conclusão demanda um estudo mais aprofundado, ratificando a necessidade de modelos hidrológicos capazes de identificar de maneira mais precisa variações sub-diárias dos processos hidrológicos.

Palavras-chave: curvas IDF; erosividade; hidrologia; regiões semiáridas

\section{Intense rainfall behavior for basin in preserved Caatinga semiarid region}

ABSTRACT: The knowledge of intense precipitation is fundamental for the planning of hydrographic basins; however, it demands robust rainfall databases, still scarce in the Brazilian semiarid region. The objective of this work was to assess the intensity duration - frequency curves (IDF) Curves in the Aiuaba Experimental Basin. Based on measurements every 5 min for 16 years (2003 to 2018), IDF curves were generated for the periods of $5 \mathrm{~min}, 30 \mathrm{~min}, 1 \mathrm{~h}, 2 \mathrm{~h}, 3 \mathrm{~h}, 6 \mathrm{~h}, 12 \mathrm{~h}, 18 \mathrm{~h}$, and $24 \mathrm{~h}$. The parameters of the Otto Pfafstetter equation for the IDF curves was calibrated as well. From the investigation, we concluded that: the IDF curves (inedited for the Inhamuns region, CE) are compatible with those of other studies in semiarid regions and indicated to be a macro-regional property; the erosivity indicated a maximum value for rainfall of duration equal to $3 \mathrm{~h}$, being this a combined effect of high intensity and high kinetic energy. This conclusion calls for further studies, ratifying the need for hydrological models capable of more accurately identifying the sub-daily variations in hydrological processes.

Key words: IDF curves; erosivity; hydrology; semiarid regions 


\section{Introdução}

Para um eficaz planejamento de uso do solo e da água de qualquer região, é imprescindível dispor de informações acerca das variáveis climáticas. Nesse contexto, as chuvas intensas são fundamentais para análises hidroambientais, como mudanças climáticas e inundações (Lu et al., 2015); secas (Araújo \& Bronstert, 2016); transporte de sedimentos e poluentes, capacidade de autodepuração dos corpos hídricos e dimensionamento de obras hídricas. Sobretudo na região semiárida do Nordeste brasileiro, que sofre com problemas de escassez hídrica em cerca de dois terços do ano, pouca profundidade e salinidade dos solos e sazonalidade do regime de chuvas (Araújo, 2012; Andrade et al., 2016; Costa \& Silva, 2017).

Apesar da relevância, os estudos das chuvas intensas esbarram em dificuldades, pois demandam contínuo monitoramento e robustos bancos de dados, o que ainda pouco ocorre em regiões tropicais semiáridas (Fechine Sobrinho et al., 2014).

Oregime pluviométrico é fundamental para o planejamento de bacias hidrográficas, inclusive para a quantificação dos impactos de ações antrópicas, como as alterações de uso do solo. Por exemplo, eventuais impactos sobre vazões fluviais podem ser avaliados com auxílio de modelos matemáticos chuva-vazão, cujas incertezas residem sobremaneira nas precipitações efetivamente ocorridas (Fadhel et al., 2017).

Outra aplicação das variáveis relacionadas ao regime pluviométrico é o estudo de erosão hídrica, ao qual pertence a erosividade da chuva (termo " $R$ " na Equação Universal de Perda de Solo). A erosividade foi modelada por Wischmeier \& Smith (1978) através de um índice que expressa a capacidade da precipitação de erodir o solo de um terreno sem cobertura vegetal. Isto é, o termo $R$ expressa o potencial da água da chuva para desagregar o solo e transportá-lo por meio do escoamento superficial subsequente. Este potencial é função de sua energia, que depende tanto do tamanho das gotas como da intensidade máxima da precipitação em 30 minutos (Wischmeier \& Smith, 1978).

Neste contexto, o objetivo principal deste estudo foi gerar curvas de intensidade-duração-frequência e analisar a erosividade da precipitação em diferentes intensidades e períodos de retorno para postos no Bioma Caatinga em bom estado de preservação. A importância deste trabalho também reside no fato de utilizar dezesseis anos de dados primários medidos a cada 5 minutos - em uma região com reconhecida carência de informações pluviográficas.

\section{Material e Métodos}

\section{Área de estudo}

O estudo foi conduzido na Bacia Experimental de Aiuaba (BEA, $12 \mathrm{~km}^{2}$ ), localizada no município homônimo, Ceará (Figura 1), tendo como coordenadas geográficas 60 34' 25" S e 40 07’ 25” W (IPECE, 2012). De acordo com a Classificação de Köppen, o clima da bacia é Bsh (semiárido com baixa latitude e altitude), com pluviosidade anual média de 562 $\mathrm{mm}$, prevalência do período chuvoso de janeiro a maio. A bacia está inteiramente inserida em uma área totalmente preservada pertencente à Estação Ecológica homônima (ESEC). A média mensal da temperatura varia de 24 a $28^{\circ} \mathrm{C}$ e a evaporação média anual em tanque Classe A é de $2500 \mathrm{~mm}$. A vegetação predominante, da Caatinga, é caracterizada por árvores de porte médio a alto, de 5 a $12 \mathrm{~m}$. A bacia possui declividade média de $19 \%$ e sua geologia é caracterizada

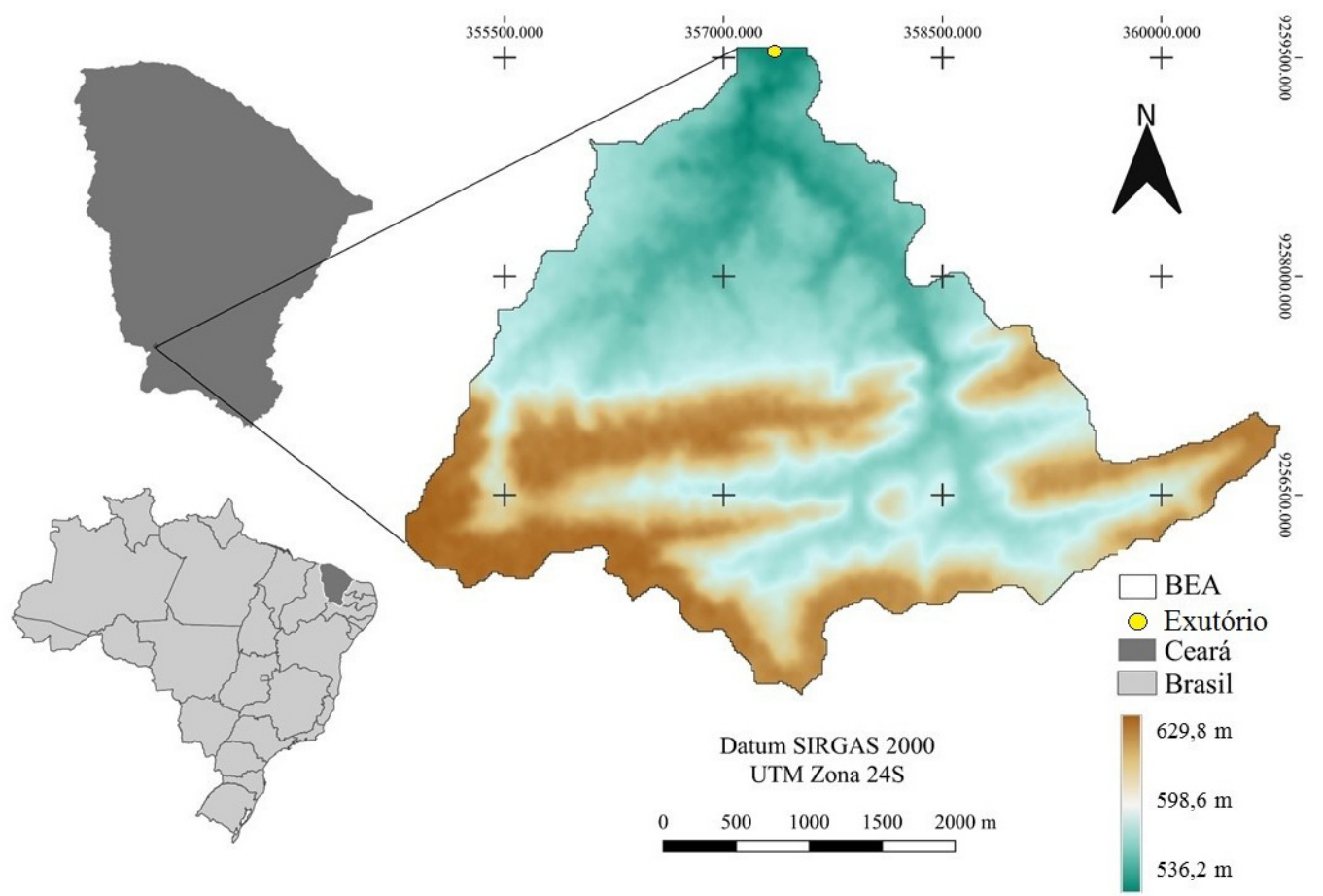

Figura 1. Mapa de localização e elevação da Bacia Experimental de Aiuaba (BEA). 
por transição entre um complexo cristalino e uma formação sedimentar (Pinheiro et al., 2016).

\section{Dados}

Duas estações pluviométricas serviram de base para este trabalho, ambas pertencentes à BEA e distantes $5 \mathrm{~km}$ entre si. O período estudado foi de 2003 a 2018, totalizando 16 anos contínuos monitorados pelo Grupo de Pesquisa Hidrossedimentológica do Semiárido (www.hidrosed.ufc.br).

Para compor as curvas foram utilizados dados de precipitação de 5 e 30 minutos, 1, 2, 3, 6, 12, 18 e 24 horas, além de chuva diária. As chuvas de duração de 30 minutos ou superiores foram compostas a partir das chuvas de 5 minutos. Os dados foram dispostos em ábacos, nos quais foram traçadas as curvas de Pfafstetter (1958), cujos parâmetros foram calibrados para a Bacia Experimental de Aiuaba. As curvas de intensidade - duração - frequência (IDF) de Pfafstetter (1958) é dada pela Equação 1 .

$$
\mathrm{i}_{\max }=\frac{\mathrm{k} \cdot \mathrm{T}_{\mathrm{r}}^{\mathrm{m}}}{\left(\mathrm{t}+\mathrm{t}_{0}\right)^{\mathrm{n}}}
$$

em que: $k, m, n, t_{0}$ são parâmetros de processos que devem, portanto, ser calibrados. Tr é o período de retorno (anos), t é a duração da chuva, em minutos.

Os parâmetros da Equação 1 foram obtidos por meio de método iterativo para sistemas de equações não-lineares (ferramenta Solver do software Microsoft Excel ${ }^{\circledR}$, que utiliza o método do Gradiente Reduzido Generalizado, GRG não-linear, descrito por Abadie \& Carpentier, 1969) cuja função objetivo foi a medida de eficiência Coeficiente de Nash e Sutcliffe - NSE (Nash \& Sutcliffe, 1970). Com o fim de avaliar os valores dos parâmetros empíricos, esta função foi adaptada para a forma da Equação 2.

$$
\mathrm{i}_{\max }=\frac{\mathrm{k} \cdot \mathrm{T}_{\mathrm{r}}^{\mu}}{\delta}
$$

em que: $\delta=\left(t+t_{0}\right)^{n}$ e $\mu=m$.

\section{Parametrização das curvas IDF e da erosividade}

Os valores de altura pluviométrica, em $\mathrm{mm}$, foram dispostos em ordem decrescente. O NSE, utilizado para calibrar os parâmetros, determina a magnitude relativa da variância residual (ruído) comparada à variação de dados medidos (informação). O NSE indica quão bem dados observados e simulados se relacionam; varia entre $-\infty$ e 1 , com NSE = 1 sendo o valor ótimo. Para valores acima de 0,70 as simulações são vistas como aceitáveis, enquanto valores $<0,0$ indicam que a média das observações é um melhor indicador do que os valores simulados, o que indica desempenho inaceitável (Nash \& Sutcliffe, 1970). O coeficiente foi obtido conforme a Equação 3.

$$
\mathrm{NSE}=1-\frac{\sum\left(\varnothing_{\mathrm{m}}-\varnothing_{\mathrm{c}}\right)^{2}}{\left(\varnothing_{\mathrm{m}}-\mathrm{T}_{\mathrm{m}}\right)^{2}}
$$

em que: $\emptyset_{m}$ é o valor medido, $\emptyset_{c}$ é o valor calculado e $T_{m}$ é a média dos valores medidos.

O cálculo da energia cinética da chuva foi realizado por meio do método proposto por Wischmeier \& Smith (1978) conforme a Equação 4.

$$
\mathrm{E}_{\mathrm{c}}=\mathrm{H}\left(11,9+8,73 \cdot \log _{10} \mathrm{I}\right)
$$

em que: $E_{c}$ é a energia cinética da chuva $\left(M J \mathrm{ha}^{-1} \mathrm{~mm}^{-1}\right)$ e I é a intensidade da chuva $\left(\mathrm{mm} \mathrm{h}^{-1}\right)$.

Com a determinação da Energia Cinética obtém-se então a erosividade por meio da Equação 5, na qual $\mathrm{R}$ é a erosividade da chuva (MJ.ha ${ }^{-1} \cdot \mathrm{mm} \cdot \mathrm{h}^{-1}$ ); $\mathrm{I}_{30}$ é a intensidade máxima da chuva em $30 \mathrm{~min}\left(\mathrm{~mm} \cdot \mathrm{h}^{-1}\right)$; e $\mathrm{E}_{\mathrm{c}}$ é a energia cinética da chuva $\left(\mathrm{MJ}_{\text {.ha-1 }}{ }^{-1}\right)$.

$$
\mathrm{R}=\sum\left(\mathrm{I}_{30} \cdot \mathrm{E}_{\mathrm{c}}\right)
$$

\section{Resultados e Discussão}

Na Figura 2 se verifica que para cada período de retorno (Tr), a intensidade decresce quando o intervalo de duração $t$ cresce. Evidenciou-se o decréscimo da intensidade acompanhando o aumento na duração das chuvas, caracterizando comportamento inversamente proporcional. A função densidade de probabilidade de melhor ajuste, como determinado por Araújo \& Piedra (2009) na mesma bacia é a de Gumbel. Os parâmetros apresentaram bom ajuste, com coeficiente de determinação $\left(R^{2}\right)$ superiores a $90 \%$ e NSE classificado como muito bom (Nash \& Sutcliffe, 1970). O comportamento das famílias de curvas com concavidade para baixo são observados em trabalhos anteriores feitos para o Ceará (Sobrinho et al., 2014) outras regiões brasileiras (Lorenzoni et. al, 2014; Souza et al., 2012) e também em regiões áridas ou semiáridas do globo (Elsebaie, 2012; Rodríguez et al., 2014; Bairwa et al., 2016).
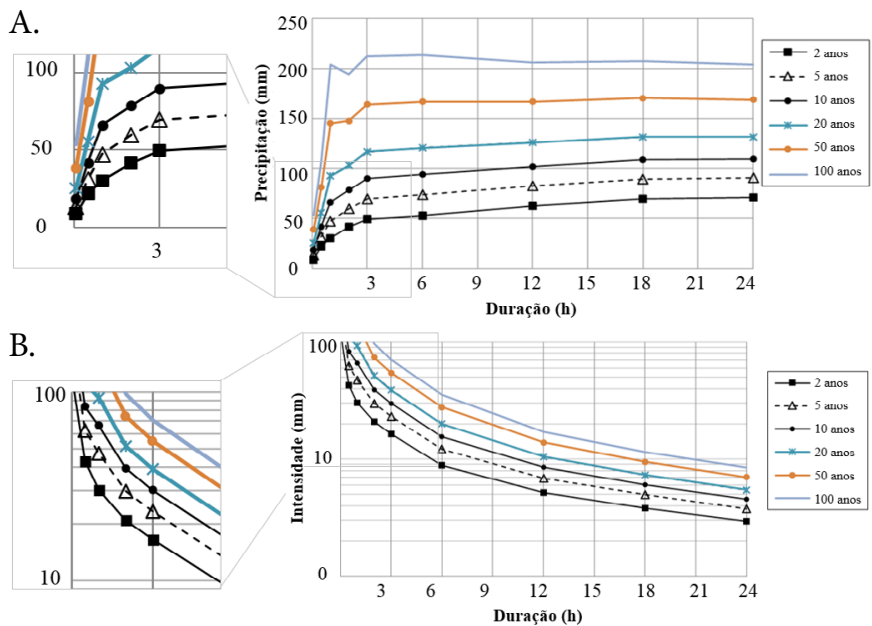

Figura 2. Síntese das curvas IDF para a Bacia Experimental de Aiuaba (2003 - 2016). (A) intensidade versus duração da precipitação e; (B) altura pluviométrica versus duração da precipitação. 
Na Tabela 1 são apresentadas as equações de intensidadeduração-frequência para a localidade estudada, com seus respectivos coeficientes NSE e na Tabela 2 as diferentes intensidades médias para Tempos de Retorno de 2 a 100 anos. Observa-se que NSE tende a diminuir com o aumento da duração da chuva. Isto está associado à facilidade, em certa medida, de previsão dos acontecimentos em chuvas de menor duração em relação a chuvas de maior duração, visto que nestas o acúmulo de incertezas nos processos hidrológicos torna-se consideravelmente superior; neste caso estudos de incertezas utilizando abordagem estatística e estocástica são indicados (Fadhel et al., 2017; Mélèse et al., 2018). Isto é, os erros advindos da estimativa das intensidades máximas para a região tendem a crescer com

Tabela 1. Equações de intensidade máxima média de precipitação pluvial (i), em $\mathrm{mm} \mathrm{h}^{-1}$, em função do período de retorno $(T)$, em anos, e respectiva duração da precipitação, calibradas para a Bacia Experimental de Aiuaba, CE.

\begin{tabular}{ccc}
\hline Duração & \multicolumn{1}{c}{ Equação } & NSE \\
\hline $5 \mathrm{~min}$ & $\mathrm{i}=\frac{3258,95 \cdot \mathrm{Tr}^{0,464}}{43,20}$ & 0,96 \\
$30 \mathrm{~min}$ & $\mathrm{i}=\frac{1419,13 \cdot \mathrm{Tr}^{0,415}}{44,31}$ & 0,95 \\
$1 \mathrm{~h}$ & $\mathrm{i}=\frac{1001,45 \cdot \mathrm{Tr}^{0,488}}{46,63}$ & 0,96 \\
$2 \mathrm{~h}$ & $\mathrm{i}=\frac{796,41 \cdot \operatorname{Tr}^{0,394}}{50,29}$ & 0,90 \\
$3 \mathrm{~h}$ & $\mathrm{i}=\frac{652,54 \cdot \operatorname{Tr}^{0,373}}{51,40}$ & 0,78 \\
$6 \mathrm{~h}$ & $\mathrm{i}=\frac{380,97 \cdot \operatorname{Tr}^{0,357}}{55,39}$ & 0,88 \\
$12 \mathrm{~h}$ & $\mathrm{i}=\frac{216,94 \cdot \operatorname{Tr}^{0,306}}{51,69}$ & 0,80 \\
$18 \mathrm{~h}$ & $\mathrm{i}=\frac{161,06 \cdot \operatorname{Tr}^{0,281}}{51,06}$ & 0,71 \\
$24 \mathrm{~h}$ & $\mathrm{i}=\frac{129,74 \cdot \operatorname{Tr}^{0,271}}{53,18}$ & 0,72 \\
\hline
\end{tabular}

o aumento da duração da precipitação pluvial. Ademais, sugere-se que as estimativas dos parâmetros calibrados nesse trabalho são pertinentes para serem adotadas em regiões de Caatinga preservada ainda não monitoradas ou com monitoramento incipiente.

Nota-se na Figura 3 que a erosividade das chuvas intensas indicou um valor máximo para chuvas de duração igual a 3 horas, iniciando a partir deste ponto um decréscimo abrupto da curva para posterior suavidade. Atribui-se isto a um efeito combinado de alta intensidade e alta energia cinética, típica das precipitações em regiões semiáridas (Oliveira et al., 2017a). Entretanto, esta conclusão demanda um estudo mais aprofundado, buscando elucidar o(s) agente(s) causadores deste comportamento a partir da duração de $3 \mathrm{~h}$. Recomenda-se que seja realizado para diversos postos pluviométricos, que disponha de valores calculados de intensidades semelhantes entre si devido à proximidade geográfica entre as estações e localização em mesma zona climatológica. A divisão em classes foi realizada de acordo com a classificação para interpretação de índice de erosividade para o Brasil (Oliveira et al., 2017b).

De fato, registros de precipitação na BEA feitos por Medeiros \& Araújo (2014) indicaram alta concentração temporal de precipitação: $51 \%$ do total de chuvas ocorridos em $10 \%$ dos dias mais chuvosos. Além disso, os autores supracitados observaram que a variabilidade nos intervalos de tempo sub-diários é bastante alta, causando chuvas intensas em intervalos de tempo curtos. Como consequência, eventos com alto poder erosivo ocorrem apesar da baixa precipitação anual. Em estudo investigativo da erosividade e de padrões hidrológicos realizado no Agreste de Pernambuco, Santos \& Montenegro (2012) não encontraram correlação significativa entre erosividade e duração da precipitação.

Tabela 2. Intensidade e altura pluviométrica segundo equação de Pfafstetter para diferentes durações (5min a 24h) e períodos de retorno (2 a 100 anos) na Bacia Experimental de Aiuaba, CE.

\begin{tabular}{|c|c|c|c|c|c|c|}
\hline \multirow{2}{*}{$\begin{array}{l}\operatorname{Tr} \text { (anos)/ } \\
\text { Duração }\end{array}$} & \multicolumn{2}{|c|}{2} & \multicolumn{2}{|c|}{5} & \multicolumn{2}{|c|}{10} \\
\hline & $\mathrm{i}(\mathrm{mm} / \mathrm{h})$ & $\mathrm{H}(\mathrm{mm})$ & $i(\mathrm{~mm} / \mathrm{h})$ & $\mathrm{H}(\mathrm{mm})$ & $\mathrm{i}(\mathrm{mm} / \mathrm{h})$ & $\mathrm{H}(\mathrm{mm})$ \\
\hline $5 \mathrm{~min}$ & 104,09 & 8,67 & 159,31 & 13,28 & 219,80 & 18,32 \\
\hline $30 \mathrm{~min}$ & 42,72 & 21,36 & 62,51 & 31,26 & 83,37 & 41,69 \\
\hline $2 \mathrm{~h}$ & 20,80 & 41,60 & 29,84 & 59,67 & 39,20 & 78,39 \\
\hline $3 \mathrm{~h}$ & 16,44 & 49,33 & 23,15 & 69,44 & 29,98 & 89,94 \\
\hline $6 \mathrm{~h}$ & 8,81 & 52,85 & 12,22 & 73,31 & 15,65 & 93,89 \\
\hline $18 \mathrm{~h}$ & 3,83 & 68,99 & 4,96 & 89,25 & 6,03 & 108,45 \\
\hline $24 \mathrm{~h}$ & 2,94 & 70,63 & 3,77 & 90,49 & 4,55 & 109,16 \\
\hline \multirow{2}{*}{$\begin{array}{c}\operatorname{Tr} \text { (anos)/ } \\
\text { Duração }\end{array}$} & \multicolumn{2}{|c|}{20} & \multicolumn{2}{|c|}{50} & \multicolumn{2}{|c|}{100} \\
\hline & $\mathrm{i}(\mathrm{mm} / \mathrm{h})$ & $\mathrm{H}(\mathrm{mm})$ & $i(\mathrm{~mm} / \mathrm{h})$ & $\mathrm{H}(\mathrm{mm})$ & $i(\mathrm{~mm} / \mathrm{h})$ & $\mathrm{H}(\mathrm{mm})$ \\
\hline $5 \mathrm{~min}$ & 303,27 & 25,27 & 464,13 & 38,68 & 640,39 & 53,37 \\
\hline $30 \mathrm{~min}$ & 111,20 & 55,60 & 162,72 & 81,36 & 217,03 & 108,51 \\
\hline $6 \mathrm{~h}$ & 20,04 & 120,26 & 27,80 & 166,80 & 35,61 & 213,64 \\
\hline $12 \mathrm{~h}$ & 10,50 & 126,01 & 13,90 & 166,81 & 17,19 & 206,24 \\
\hline $18 \mathrm{~h}$ & 7,32 & 131,78 & 9,47 & 170,48 & 11,51 & 207,15 \\
\hline $24 \mathrm{~h}$ & 5,49 & 131,67 & 7,03 & 168,71 & 8,48 & 203,51 \\
\hline
\end{tabular}




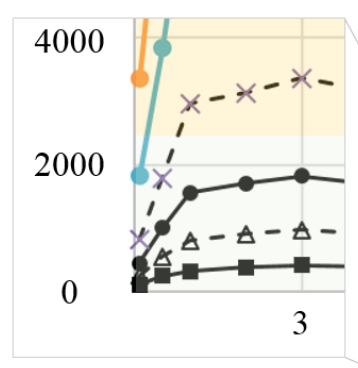

Erosividade baixa $(\mathrm{R} \leq 2452)$

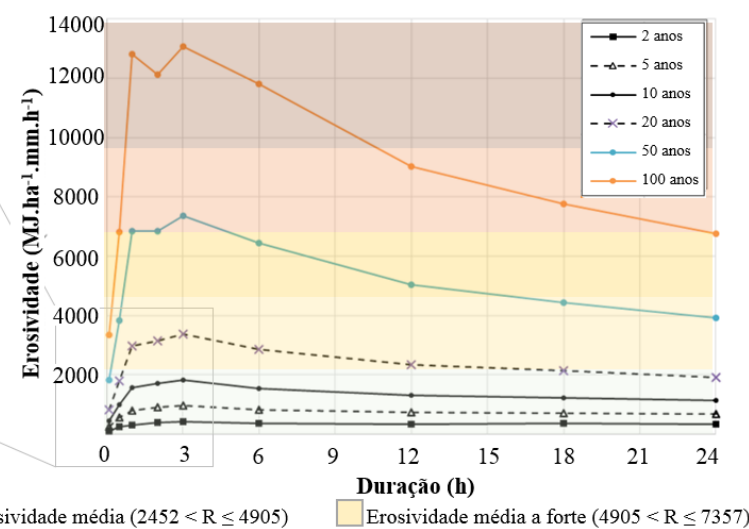

Erosividade muito forte $(\mathrm{R}>9810)$

Figura 3. Erosividade (MJ.ha-1 $\cdot \mathrm{mm} \cdot \mathrm{h}^{-1}$ ) das chuvas segundo equação de Wischmeier e Smith (1978) e classes segundo Oliveira et al. (2017b).

Tabela 3. Fator Erosividade (R) da USLE (MJ ha-1 $\mathrm{mm} \mathrm{h}^{-1}$ ) e respectivas durações das chuvas segundo equação de Wischmeier e Smith (1978) para a Bacia Experimental de Aiuaba, CE.

\begin{tabular}{ccccccc}
\hline Tr (anos)/ & $\mathbf{2}$ & $\mathbf{5}$ & $\mathbf{1 0}$ & $\mathbf{2 0}$ & $\mathbf{5 0}$ & $\mathbf{1 0 0}$ \\
\cline { 2 - 7 } Duração & \multicolumn{5}{c}{$\mathbf{R}\left(\mathbf{M J ~ h a}^{-1} \mathbf{m m ~ h}^{-1} \mathbf{)}\right.$} \\
\hline $5 \mathrm{~min}$ & 107.01 & 240.54 & 442.76 & 814.79 & 1824.70 & 3357.88 \\
$30 \mathrm{~min}$ & 242.04 & 548.55 & 996.44 & 1787.54 & 3837.46 & 6827.72 \\
$1 \mathrm{~h}$ & 313.57 & 795.88 & 1555.93 & 2969.27 & 6841.20 & 12806.14 \\
$2 \mathrm{~h}$ & 384.17 & 906.31 & 1702.66 & 3137.92 & 6843.19 & 12140.25 \\
$3 \mathrm{~h}$ & 417.62 & 973.65 & 1824.33 & 3367.43 & 7373.51 & 13083.95 \\
$6 \mathrm{~h}$ & 351.20 & 809.32 & 1522.37 & 2852.42 & 6458.08 & 11811.17 \\
$12 \mathrm{~h}$ & 342.81 & 730.94 & 1303.47 & 2332.18 & 5041.81 & 9020.67 \\
$18 \mathrm{~h}$ & 346.54 & 708.67 & 1224.96 & 2127.17 & 4436.32 & 7755.48 \\
\hline $24 \mathrm{~h}$ & 331.10 & 662.13 & 1125.25 & 1921.88 & 3926.91 & 6771.33 \\
\hline
\end{tabular}

\section{Conclusões}

Os dados apresentados, inéditos para a região dos Inhamuns, Ceará, demonstraram valores compatíveis com os de outros estudos na região semiárida do Brasil.

As curvas IDF (intensidade-duração-frequência) indicaram ser uma propriedade macrorregional, o que permite o uso de curvas de postos da mesma região para estudos que demandem precisão limitada.

Dentro da macrorregião do Ceará, as características das chuvas intensas indicam ser uma função mais sensível da altitude que de normais climatológicas, como chuva média anual, temperatura ou evaporação potencial.

A erosividade das chuvas intensas indicou um valor máximo para chuvas de duração igual a 3 horas, sendo esse um efeito combinado de alta intensidade e alta energia cinética. Esta conclusão demanda um estudo mais aprofundado, que se recomenda seja realizado para diversos postos pluviométricos, ratificando a necessidade de modelos hidrológicos capazes de identificar de maneira mais precisa variações sub-diárias dos processos hidrológicos.

\section{Agradecimentos}

O presente trabalho foi realizado com apoio da Coordenação de Aperfeiçoamento de Pessoal de Nível Superior - Brasil (CAPES) - Código de Financiamento 001.

\section{Literatura Citada}

Abadie, J.; Carpentier, J. Generalization of the Wolfe reduced gradient method to the case of nonlinear constraints. In: Fletcher, R. (Ed.). Optimization. New York: Academic Press, 1969. p.37-47.

Andrade, E. M.; Sena, M. G. T.; Silva, A. G. R.; Pereira, F. J. S.; Lopes, F. B. Uncertainties of the rainfall regime in a tropical semi-arid region: the case of the State of Ceará. Revista Agro@mbiente Online, v. 10, n. 2, p.88-95, 2016. https://doi.org/10.18227/19828470ragro.v10i2.3500.

Araújo, J. C. Recursos hídricos em regiões semiáridas. In: Gheyi, H. R.; Paz, V.P. da S.; Medeiros, S. de S.; Galvão, C. de O. (Eds.). Recursos hídricos em regiões semiáridas. Estudos e aplicações. 1ed. Cruz das Almas: Instituto Nacional do Semiárido, 2012. p. 30-43. https://portal.insa.gov.br/acervo-livros/195-recursos-hidricosem-regioes-semiaridas-estudos-e-aplicacoes. 25 Ago. 2018.

Araújo, J. C.; Bronstert, A. A method to assess hydrological drought in semi-arid environments and its application to the Jaguaribe River basin, Brazil. Water International, v.41, n.2, p.213-230, 2016. https://doi.org/10.1080/02508060.2015.1113077.

Araújo, J. C.; Piedra, J. I. G. Comparative hydrology: analysis of a semiarid and a humid tropical watershed. Hydrological Processes, v. 23, p. 1169-1178, 2009. https://doi.org/10.1002/hyp.7232.

Bairwa, A. K.; Khosa, R.; Maheswaran, R. Developing intensity duration frequency curves based on scaling theory using linear probability weighted moments: A case study from India. Journal of Hydrology, v.542, p.850-859, 2016. https://doi.org/10.1016/j. jhydrol.2016.09.056. 
Costa, J. A.; Silva, D. F. Distribuição espaço-temporal do Índice de anomalia de chuva para o Estado do Ceará. Revista Brasileira de Geografia Física, v.10, n.4, p.1002-1013, 2017. https://doi. org/10.26848/rbgf.v10.4.p1002-1013.

Elsebaie, I. H. Developing rainfall intensity-duration-frequency relationship for two regions in Saudi Arabia. Journal of King Saud University - Engineering Sciences, v. 24, p.131-140, 2012. https:// doi.org/10.1016/j.jksues.2011.06.001.

Fadhel, S.; Rico-Ramirez, M. A.; Han, Dawel. Uncertainty of Intensity - Duration - Frequency (IDF) curves due to varied climate baseline periods. Journal of Hydrology, v. 547, p.600-612, 2017. https:// doi.org/10.1016/j.jhydrol.2017.02.013.

Fechine Sobrinho, V. F.; Rodrigues, J. O.; Mendonça, L. A. R.; Andrade, E. M. de; Tavares, P. R. L. Desenvolvimento de equações IntensidadeDuração-Frequência sem dados pluviográficos em regiões semiáridas. Revista Brasileira de Engenharia Agrícola e Ambiental, v.18, n.7, p.727734, 2014. https://doi.org/10.1590/\$1415-43662014000700009.

Instituto de Pesquisa e Estratégia Econômica - IPECE. Perfil básico municipal, 2012. http://www.ipece.ce.gov.br/publicacoes/ perfil_basico/pbm-2012/Aiuaba.pdf. 30. Jul. 2018.

Lorenzoni, M. Z.; Prado, G do; Souza, A. H. C. de; Rezende, M. K. A.; Mioto, L. S. Curvas intensidade-duração-frequência de chuvas intensas de Cidade Gaúcha e Guaporema - PR. In: Congresso Brasileiro de Engenharia Agrícola, 14., 2014, Campo Grande. Anais... Campo Grande: SBEA, 2014. http://conbea14.sbea.org. br/2014/anais/R0391-1.pdf. 19. Jul. 2018.

Lu, Y.; Qin, X.S.; Xie, Y.J. An integrated statistical and data-driven framework for supporting flood risk analysis under climate change. Journal of Hydrology, v.533, p. 28-39, 2015. https://doi. org/10.1016/j.jhydrol.2015.11.041.

Medeiros, P. H. A.; Araújo, J. C. Temporal variability of rainfall in a semiarid environment in Brazil and its effect on sediment transport processes. Journal of Soils and Sediments, v. 14, n. 7, p.1216-1223, 2014. https://doi.org/10.1007/s11368-013-0809-9.

Mélése, V.; Blanchet, J.; Molinié, G. Uncertainty estimation of Intensity-Duration-Frequency relationships: A regional analysis. Journal of Hydrology, v.558, p.579-591, 2018. https://doi. org/10.1016/j.jhydrol.2017.07.054.
Nash, J. E.; Sutcliffe, J. V. River flow forecasting through conceptual models: Part I - A discussion of principles. Journal of Hydrology, v. 10, n.3, p. 282-290, 1970. https://doi.org/10.1016/00221694(70)90255-6.

Oliveira, P. T. S.; Wendland, E.; Nearing, M. A. Rainfall erosivity in Brazil: A review. Catena, v. 100, p.139-147, 2017b. https://doi. org/10.1016/j.catena.2012.08.006.

Oliveira, P. T.; Silva, C. M. S.; Lima, K. C. Climatology and trend analysis of extreme precipitation in subregions of Northeast Brazil. Theoretical and Applied Climatology, v. 130, n.1-2, p.7790, 2017a. https://doi.org/10.1007/s00704-016-1865-z.

Pfafstetter, O. Chuvas intensas no Brasil. 2.ed. Rio de Janeiro: Departamento Nacional de Obras e Saneamento, 1958. 426 p.

Pinheiro, E. A. R.; Metselaar, K.; Lier, Q. de J. van; Araújo, J. C. de. Importance of soil - water to the Caatinga biome, Brazil. Ecohydrology, v. 9, n. 7, p.1313-1327, 2016. https://doi. org/10.1002/eco.1728.

Rodríguez, R.; Navarro, X.; Casas, M. C.; Ribalaygua, J.; Russo, B.; Pouget, L.; Redaño, A. Influence of climate change on IDF curves for the metropolitan area of Barcelona (Spain). International Journal of Climatology, v. 34, n.3, p.643-654, 2014. https://doi. org/10.1002/joc.3712.

Santos, T. E. M. dos; Montenegro, A. A. A. Erosividade e padrões hidrológicos de precipitação no Agreste Central pernambucano. Revista Brasileira de Engenharia Agrícola e Ambiental. v. 16, n. 8, p. 871-880, 2012. https://doi.org/10.1590/S141543662012000800009.

Souza, R. O. R. de M.; Scaramussa, P. H. M.; Amaral, M. A. C. M. do; Neto, J. A. P.; Pantoja, A. V.; Sadeck, L. W. R. Equações de chuvas intensas para o estado do Pará. Revista Brasileira de Engenharia Agrícola e Ambiental, v.16, n.9, p.999-1005, 2012. https://doi. org/10.1590/S1415-43662012000900011.

Wischmeier, W.H.; Smith, D.D. Predicting rainfall erosion losses - a guide to conservation planning. Washington: USDA, 1978. 58p. (USDA. Agriculture Handbook, 537). https://naldc.nal.usda.gov/ download/CAT79706928/PDF. 28 Jul. 2018. 